\title{
Complete Methods for Building Complete Applications
}

\author{
Naveen Prakash \\ 1 Knowledge Park Phase II, Greater NOIDA 201306, lndja \\ praknav@hotmail.com
}

\begin{abstract}
ISDMs) produce a product, the application, by following a development process model. We argue that in failing to produce the application process model that supports the application product, ISDMs only address part of the IS development problem. Additionally, we show that there is, in fact, a range of abstractions of the application product and process and ISDMs do not build these abstractions.. To address these jssues, we define the completeness principle that integrates the $100 \%$ and conceptualization principles of conceptual modelling. This principle states that an information system should be a faithful representation of the product and process models at the required level of conceptualization. A method that complies with this principle is called a complete method. We develop a complete method and discuss issues raised in developing such methods.
\end{abstract}

\section{Introduction}

The development of products embedded in business is an issue taken up by the Information Systems community. We have put in considerable effort in product development and in modelling IS development processes for producing products. The approach to product development is to build a schema, given an application and a data model. Thus, for example, for the Oberoi Hotels Reservation System an Oberoi schema in ER form is built. Several process models have been built to define the process to be followed in constructing such schemata. Two points are to be noticed:

In developing an application product, our concern is the immediate application and not the larger domain of which the application is a part. Thus we develop the 
Oberoi Hotel schema but not a generic hotel reservation schema. Extending this, we find that there is in fact a range of abstractions above the Oberoi Hotel schema: the hotel reservation schema, the more abstract 'reservation' schema, the even more abstract resource allocation schema and so on. Yet the IS development process aims to develop an application product and not the range of schema abstractions as products.

Interest in process models is centred on our problem of product development. We investigate process models that help $u s$ in solving our problem of developing schemata. However, there is an application views point that we totally ignore. To illustrate, consider a hotel reservation schema and assume that it meets its requirements. When installed in an organization, this schema is instantiated for the different hotel booking requests. Let the hotel be a transit hotel that gives bookings for one day only. Then, upon getting a reservation request, we can instantiate the schema components in two different orders. We refer to a schema instantiation order as an Application Process Model (APM). The two APMs in our example are shown in Table 1 .

Table I: Two Application Process Models

\begin{tabular}{|l|l|}
\hline APM1 & APM2 \\
\hline Requestor (name, address) & Booking(date, price) \\
\hline Booking(date, price) & Availability(number of rooms, type of room) \\
\hline $\begin{array}{l}\text { Availability(number of rooms, } \\
\text { type of room) }\end{array}$ & Requestor (name, address) \\
\hline
\end{tabular}

The application process models of Table I show two strategies for room reservation. APM1 considers booking issues like availability, prices etc. only after all requestor information is obtained whereas APM2 considers booking issues first and thereafter, once the booking is notionally done, considers requestor information to complete it. Evidently, these constitute two different ways of doing business. The APM selected should be the one that best fits into the business process in which the reservation system is embedded.

The foregoing shows an APM plays an important role in the success of an IS product. Yet the IS community ignores these. Consequently, methods that produce an APM have not been engineered

We believe that complete application development cannot be restricted to development of application products only but must also have the capability to support the range of schema abstractions and associated APMs. We propose the completeness principle that states that an information system should be a faithful representation of the product and process models at the required level of conceptualization. A diagrammatic representation of this principle is shown in Fig. 1. As shown, at abstraction level $\mathrm{i}, 0 \leq \mathrm{i} \leq \mathrm{n}$, both the APM and product models are to be represented.

The completeness principle subsumes both the 100\% and conceptualization principles (Gri82, Hoe94) of conceptual modelling. According to the $100 \%$ principle, everything that is relevant should be represented, and the representation should contain only that which is relevant. Traditionally, this principle has been applied to product aspects only and the completeness principle here proposes to 
extend it to the process aspects of applications as well: when developing the product model of an application, one should develop the APM as well. This is shown in the boxes of Fig. 1 and implies that an application is the integration of the product and application process models.

The conceptualization principle says that the representation should be independent of any implementation issues. In conceptual modelling, this has been interpreted to mean independence of the conceptual schema from the underlying implementation platform. The completeness principle proposed here extends this to cover every pair of abstraction levels of the representation. This is shown on the left side of Fig. 1. Consider abstractions at level $n$ and level (n-1). The completeness principle treats level (n-1) as the 'implementation platform' of the abstraction at level $n$. When $n=1$ then we get the conceptual modelling view of the conceptualization principle: the schema at the first level is the conceptual schema whereas the $0^{\text {th }}$ level is its implementation platform.

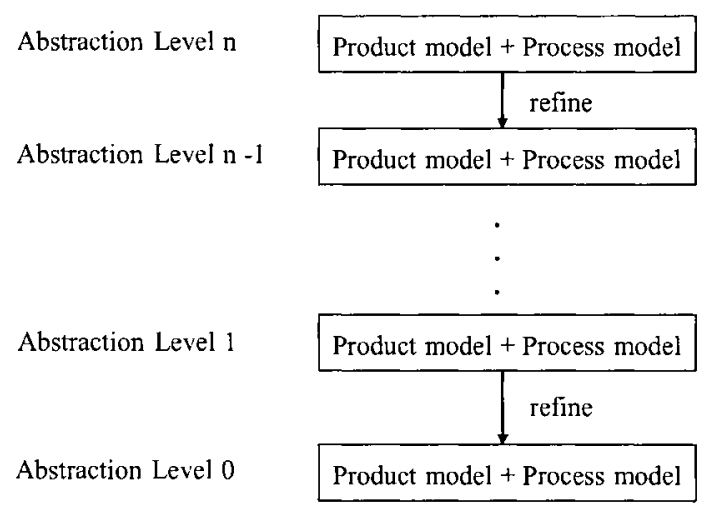

Fig. 1: The completeness principle

Taken as a whole, the completeness principle says that applications can be visualized at different levels of abstraction and at each level modelling is complete if and only if both the application product and application process models have been developed. The application at level (n-1) is a refinement of its abstraction at level $\mathrm{n}$.

In this paper, we consider the impact of the completeness principle on Information Systems Development Methods (ISDM). We shall refer to an ISDM that complies with the completeness principle as a complete method. That is, a complete method is one that provides capability to develop both the product and associated application process model at the stated level of conceptualization. We show that method engineering approaches that integrate the product and process aspects of methods have the capacity to build complete methods. In doing this, we use the generic method model (Pra06). This model treats a method as a triple $<\mathrm{M}$, Dep, E $>$ where $M$ is a set of method blocks, Dep is a set of dependencies between these methods, and $E$ is an enactment mechanism. $M$ and Dep can be organized in a dependency graph. This graph is the set of all routes that can be followed in the method; it is the set of all process models that can be built by performing a walk through the dependency graph. 
The layout of this paper is as follows. In the next section we consider the impact of the completeness principle on IS development. In section III contains a brief overview of the generic model that highlights those features that we shall use here. In section IV we illustrate the completeness principle by taking the example of issuing permits. We build product and process models at two abstraction levels. In section $\mathrm{V}$, we outline a tool that takes process information as input and produces the process model for a product. In section VI, we consider related work and show how the completeness principle brings new issues to the forefront.

\section{Implication of the Completeness Principle}

The completeness principle implies that information systems can be developed in two ways

1. By extending the scope of Information Systems Development Methods to cover systems at all levels of abstraction.

2. By starting with an abstraction at level $n$ and refining it to construct abstraction level ( $n-1)$ recursively till the desired level of abstraction is reached. in turn.

Though the concern in this paper is the former, we briefly consider each of these

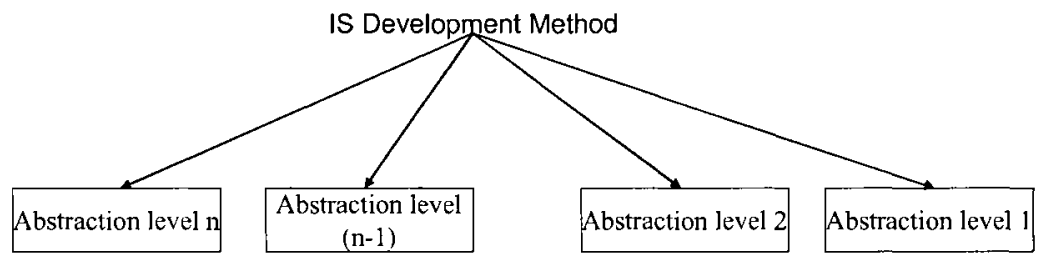

Fig. 2: Scope of an IS Development Method

Fig. 2 shows the scope of an ISDM. Whereas today, an ISDM builds only abstraction level 1, the completeness principle says that it should have the capability to build any level. An example of this is shown in Fig. 3.

$\begin{array}{ll}\text { Meta domain model } & \begin{array}{l}\text { Resource } \\ \text { allocation }\end{array} \\ \text { Application Domain } & \begin{array}{l}\text { Reservation } \\ \text { domain }\end{array} \\ \text { model } & \text { Oberoi, Taj } \\ \text { Application } & \text { reservation }\end{array}$

Fig. 3: Example of Levels of Abstraction

On the left hand side of this Figure, we see three abstraction levels. The lowest is the Application layer, the next higher is the Application Domain layer and the 
highest is the Meta domain layer. On the right hand side is an example of each of these. As shown, the lowest layer is inhabited by the Oberoi Hotels application; its domain is the Reservation domain at the next higher level and finally, its meta domain is that of Resource Allocation. The completeness principle says that an ISDM should be able to develop all the models on the right hand side of Fig. 3.

The second implication of the completeness principle is illustrated in Fig. 4. As shown, the Meta layer is converted to the domain layer through a domain development process and the latter is in turn converted to the application by an application development process.

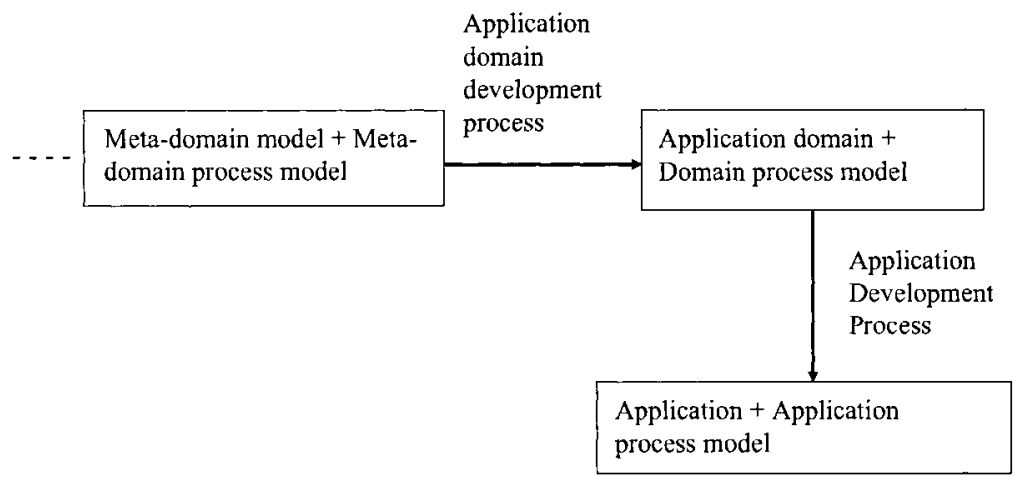

Fig. 4: Progressing through Meta Levels

This is exemplified in Fig. 5. As shown, the resource allocation meta domain is converted to the reservation domain by the reservation domain development process which, in turn, is converted to the application proper by the hotel reservation development process.

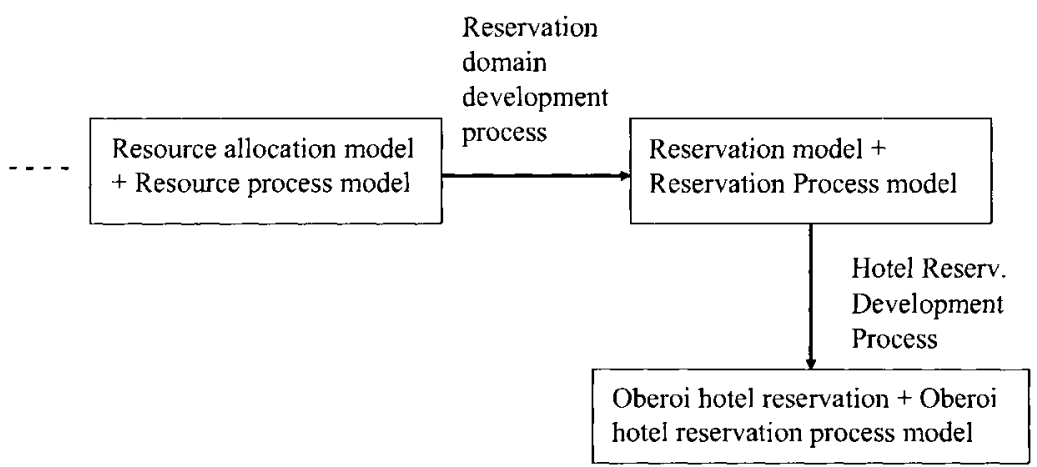

Fig. 5: Building an Application through Progression

As mentioned earlier, the focus of this paper is on elaborating the scope of an ISDM and the second approach indicated here shall be the subject of another paper. 


\section{The Generic Method Model}

The generic method model was developed (Pra06) as means to capture the essential nature of methods, devoid of any commitment to meta concepts as in metamodels. The generic method model integrates product and process aspects together. We shall provide only a broad view of the generic model so as to show its capability to meet the completeness criterion. For full details of the generic model please refer to (Pra06). However, it must be noted that we have extended the generic model to explicitly represent the relationship of process primitives with the process model as shown in Fig. 6.

According to the generic view, a method is a triple $<M, D, E>$ where $M$ is the set of method blocks, $D$ is the set of dependencies between method blocks, and $E$ is the enactment mechanism. The notion of a dependency is used to build a dependency graph with nodes as method blocks and edges as dependency types. A dependency graph has START nodes that have no edges entering them and STOP nodes that have no edges leaving them. The enactment algorithm guides method enactment from START nodes through the intermediate nodes to STOP nodes.

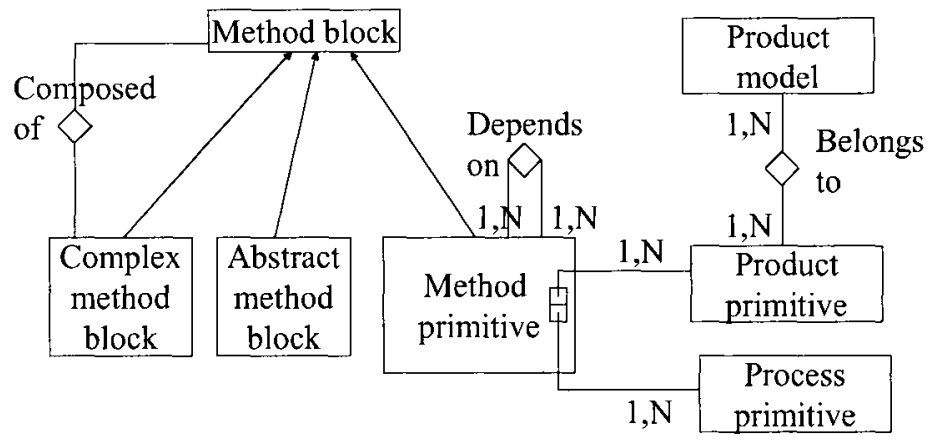

Fig. 6: The Generic Model

The generic model (Pra06) displays class independence. That is, the generic model is independent of any meta-model: the holistic structure of the artefact, its component parts and inter-relationships between these components must be expressed in abstractions high enough to instantiate a meta-model. These concepts of the generic model are themselves not technical artifacts, graphs, relationships, objects, fragments, chunks and the like. Instead, generic concepts should directly capture the notions of product and process. This is done through the notion of product and process primitives explained below.

As shown in Fig. 6, there are three kinds of method blocks, complex ones composed out of other simpler ones, abstract ones that are generalizations/specializations of method blocks and method primitives that are atomic method blocks. A method primitive is a pair <product primitive, process primitive $>$. Extending this to all method blocks, a method block can be considered as having two parts, an argument part and an action part where the action part is capable of manipulating the argument in the desired way. These two parts correspond to product primitive and process primitive respectively of Fig. 6. For 
example, <entity, attribute, attach> is a method block. It specifies that an attribute can be attached to an entity. In order for a method block to be enacted, it is necessary that instances of arguments are available. In our example, instances of attribute attributes and entity to be attached to one another must be available in the product under construction. In order to handle method blocks like <entity, create> a special product instance called 'don't care' is postulated that exists in all products. Such method blocks assume the availability of 'don't care'.

Product primitives have their origin in the product model and depending on the abstraction level and the nature of the product to be developed. Process primitives originate from the process model, and capture the kinds of operations one wants to do in the application. For a corporate system these may be Recruit and Promote for operating on Employee and Allot Budget for Department.

Table 1: Types of Dependencies

\begin{tabular}{|l|l|l|l|}
\hline Type & Urgency & Necessity & Abbreviation \\
\hline 1 & Immediate & Must & IM \\
\hline 2 & Immediate & Can & IC \\
\hline 3 & Deferred & Must & DM \\
\hline 4 & Deferred & Can & DC \\
\hline
\end{tabular}

Now consider the notion of a dependency. Two attributes, urgency and necessity, are associated with each dependency type. Urgency refers to the time at which the dependent method block, $\mathrm{O}_{2}$, is to be enacted. If $\mathrm{O}_{2}$ is to be enacted immediately after $\mathrm{O}_{1}$ is enacted then this attribute takes on the value Immediate. If $\mathrm{O}_{2}$ can be enacted any time, immediately or at any moment, after $\mathrm{O}_{1}$ has been enacted, then urgency takes on the value Deferred. Necessity refers to whether or not the dependent method block $\mathrm{O}_{2}$ is necessarily to be enacted after $\mathrm{O}_{1}$ has been enacted. If it is necessary to enact $\mathrm{O}_{2}$, then this attribute takes the value Must otherwise it has the value Can. This gives rise to four dependency types displayed in Table 1.

Using the notion of a dependency, a method can be organized as a dependency graph. We illustrate this by considering a method with the set of method primitives $\mathrm{O}$ $=\left\{\mathrm{O}_{1}, \mathrm{O}_{2}, \ldots, \mathrm{O}_{14}\right\}$. Let there be two dependency types IM and IC respectively. Let the following dependencies be defined:

IM dependencies

$$
\begin{array}{ll}
\mathrm{O}_{1} \rightarrow \mathrm{O}_{2} & \mathrm{O}_{1} \rightarrow \mathrm{O}_{3} \\
\mathrm{O}_{1} \rightarrow \mathrm{O}_{4} & \mathrm{O}_{1} \rightarrow \mathrm{O}_{5} \\
\mathrm{O}_{6} \rightarrow \mathrm{O}_{7} & \mathrm{O}_{6} \rightarrow \mathrm{O}_{8} \\
\mathrm{O}_{9} \rightarrow \mathrm{O}_{10} & \mathrm{O}_{9} \rightarrow \mathrm{O}_{11} \\
\mathrm{O}_{9} \rightarrow \mathrm{O}_{12} &
\end{array}
$$

\section{IC dependencies}

$\mathrm{O}_{1} \rightarrow \mathrm{O}_{6} \quad \mathrm{O}_{1} \rightarrow \mathrm{O}_{9}$

$\mathrm{O}_{6} \rightarrow \mathrm{O}_{13} \quad \mathrm{O}_{6} \rightarrow \mathrm{O}_{14}$

$$
\mathrm{O}_{6} \rightarrow \mathrm{O}_{13} \quad \mathrm{O}_{6} \rightarrow \mathrm{O}_{14}
$$

The organization of the method as a dependency graph for our method is shown in Fig 7. So as not to clutter up the Figure, only the IC dependencies are labelled. The non-labelled ones are assumed to be IM dependencies. 
To summarize, from the perspective of the completeness principle, the generic model brings two key notions, meta-model independence and dependency graph. The former contributes to product and process construction at any abstraction level. Thus, it is a matter of choosing the abstraction level of the product, determining the interesting product model concepts and carrying out the instantiation of the generic model. Similarly, process concepts comprising the process model at the same level of abstraction as the product are to be determined and instantiation performed. Thus, meta-model independence is the basis for development of product and process models at any abstraction level.

The dependency graph contributes to the construction of the process model associated with the product model. A path from START to STOP node represents a process model. In this sense, the dependency graph is the set of all process models that are permissible under the method.

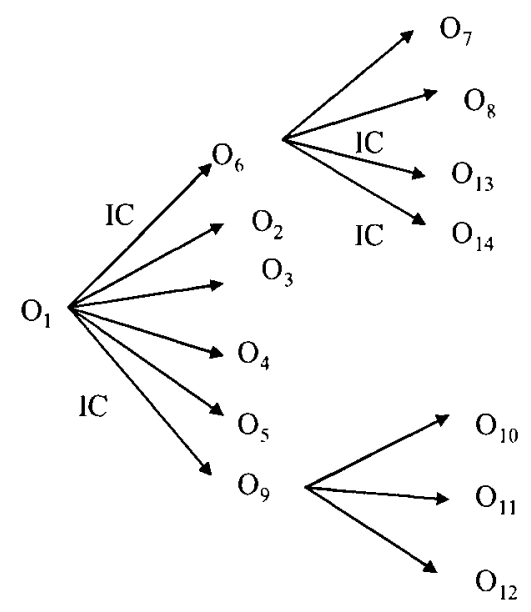

Fig. 7: A Dependency Graph

\section{Illustrating Completeness Principle}

In this section we present an example to show that the generic model follows the completeness principle: it can support the construction of a product and the associated process model at any level of abstraction. We do this by presenting a small example at different levels of abstraction as in Fig. 8. At the second abstraction level of Fig. 1, let us consider the domain of governance systems for issuing permits of different kinds, driving licenses, passports, election identity cards, running a restaurant, etc. Since all these issue permissions, we shall refer to their domain as the permit domain. We shall develop a product model and associated process model for this domain. Thereafter, at abstraction level 1, we shall consider the passport application as a refinement of the permit domain. 


$\begin{array}{ll}\text { Application Domain } & \text { Permit } \\ \text { model } & \text { domain }\end{array}$

Application

Passport

Fig. 8: The Permit Domain and its Refinement

\subsection{The Permit Domain}

In the domain of permits, a request is received for obtaining the permit. Usually, a permit is issued to an individual who may be a person, a company, a society etc. The request is processed to see that it can be further treated, that all information required is supplied, and statutory requirements are met. To check fraud or to enforce standards, verifications/inspections are carried out. There may be transaction costs associated with the issue of permits that are recovered from the requestor in the form of fees. Finally, if the requestor is found fit to receive the permit then it is issued. Permits may be lost or damaged. So, a facility for issuing duplicate permits is required. Similarly, certain permits may have to be issued on an emergency basis.

The following set of product primitives are of interest:

$P=\{$ Request, Permit, Emergency Permit, Duplicate Permit $\}$

The Process primitives are

$A=\{$ Receive, Verify, Refuse, Issue, Terminate $\}$

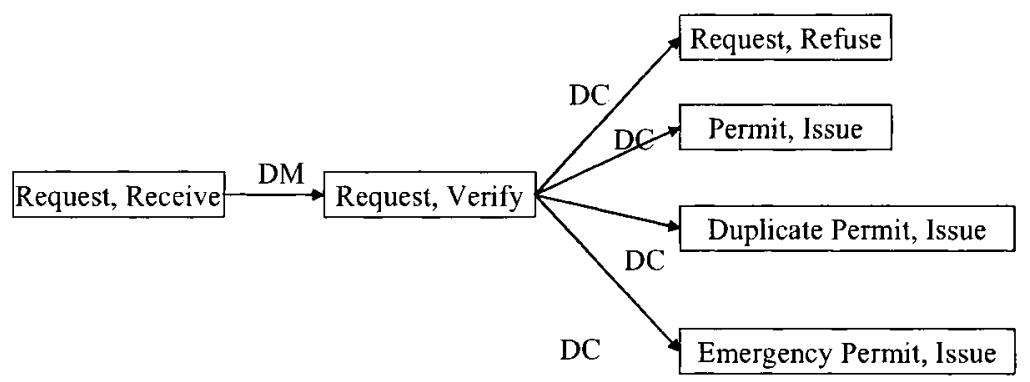

Fig. 9: The Permit Process Model

The set of method primitives is obtained by associating meaningful process types with their product types. The set of meaningful method primitives for our example is as follows:

$\mathrm{O}=\{<$ Request, Receive $>$, $<$ Request, Verify $>$, $<$ Permit, Refuse $>,<$ Permit, Issue $>$, $<$ Duplicate Permit, Issue $>$, $<$ Emergency Permit, Renew $>$, $<$ Permit, Terminate $>$ \} 
Fig. 9 shows the method primitives and dependencies of the Permit domain. The product model is not shown here. It can be developed using any standard data model like the ER. Clearly, it consists of entity types like request, permit and its specializations etc. and relationships between these.

\subsection{The Passport Application}

In this section we consider building a method for issuing Passports. The Passport Office has forms in which an application for passport services can be made. These services may be the issue of a fresh passport, renewal of an expired passport, issue of a duplicate passport, termination of a valid passport. The passport office verifies the details of the requestor and checks that there is no police record that might disallow the issue of the passport. Thereafter, the passport is issued. It is possible to add dependents of the passport holder on his/her passport or to delete them from the passport. The set of product primitives, $P$, is as follows:

$\mathrm{P}=\{$ Request, Passport, Expired passport, Duplicate Passport, Dependent $\}$

The set of process primitives, $A$, is

$A=\{$ Receive, Verify, Refuse, Issue, Renew, Terminate, Add, Delete $\}$

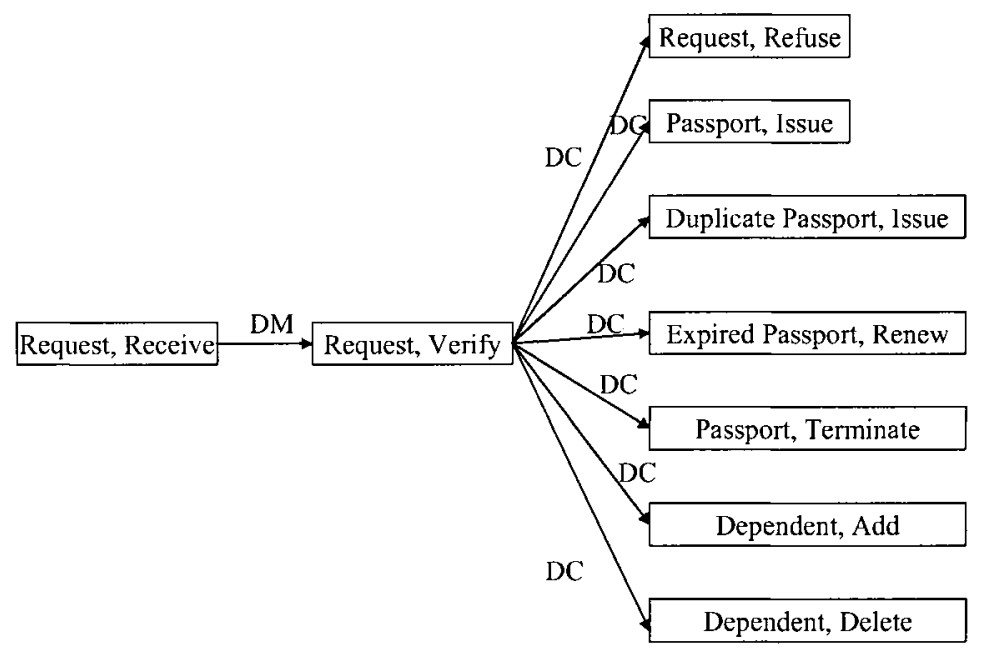

Fig. 10: The Passport Process Model

The set of method primitives is as follows:

$\mathrm{O}=\{<$ Request, Receive $>,<$ Request, Verify $>,<$ Request, Refuse $>,<$ Passport, Issue $>$, <Duplicate Passport, Issue $>$, <Expired Passport, Renew $>$, <Passport, Terminate $>,<$ Dependent, Add $>,<$ Dependent, delete $>$ \}

Fig. 10 shows the dependencies as well as the set of method primitives organized as a process model. Again, as for the permit domain, the product model is not shown here. 


\section{A Complete CASE Tool}

Information Systems Development methods present user interfaces in their CASE tools. Regarding the process model adopted by these tools, at least three attitudes exist:

1. Assume a process model. Indeed, many methods and their CASE tools assume the Linear, Cartesian one.

2. Prescribe the set of process models that can be adopted. The application engineer selects the needed one from this assembly.

3. Make no commitment to a process model. The method provides the basic structure for the application engineer to follow any process model.

Notice that all these continue to work in the case of complete methods as well.

We illustrate a CASE tool interface that obtains dependency information during application engineering. This interface (see Fig. 11) has been designed for the generic method model introduced earlier.

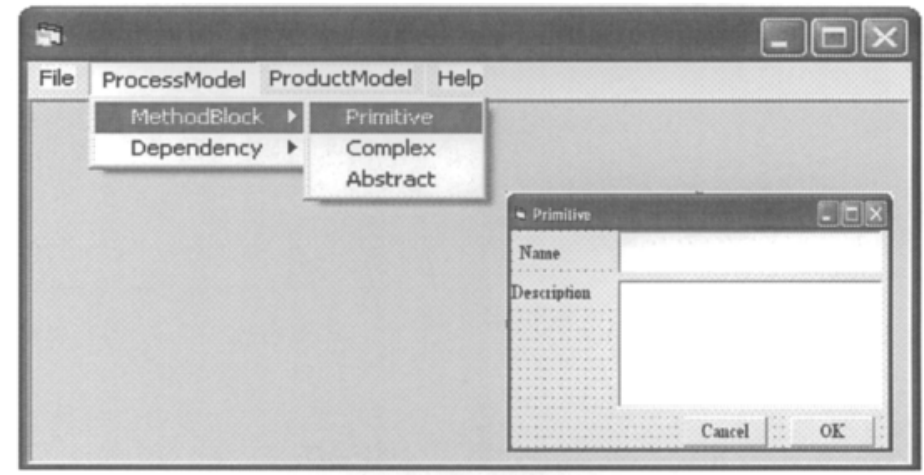

Fig. 11: Defining Method Blocks

This interface is similar to that of MetaEdit (Met93) and uses a menu bar that consists of combo boxes. As shown in the Figure, there are two menu items called Product Model and Process Model respectively. The combo box of the former contains concepts used for developing the product. Since it well understood, we do not consider this aspect here and elaborate the Process Model combo box that helps us develop the process model.

In order to produce the process model, information about method blocks and dependency information must be captured. This is achieved by the Process Model option. Clicking on the Process Model option causes the model components, Method Block and Dependency to be displayed (Fig. 11). Clicking further on method blocks enables selection of the kind of method block desired, method primitive, abstract or complex method block. Selection of these enables a pop-up box that asks for the name, description etc. of the method block. In case of abstract and complex method blocks, information about the components and specialized method blocks respectively is also asked for. 


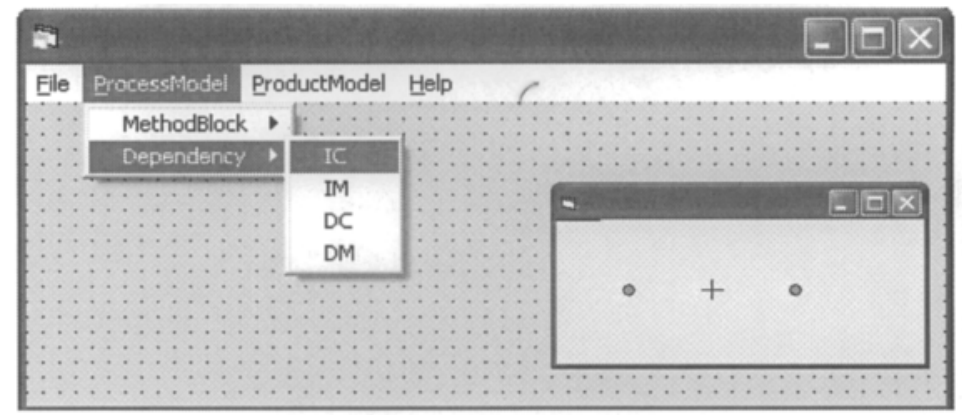

Fig. 12: Defining Dependencies between Method Blocks

Clicking on the Dependency choice (Fig. 12) invokes two capabilities. First, a cursor is enabled that is used to select two method blocks, the first of which is the source of the dependency and the second is its sink. Thus, the direction of the dependency is displayed. Second, the application engineer is asked to make a selection of the dependency type, DM, DC etc. from the displayed combo box.

\section{Comparison and Discussion}

In this section we consider the implications of developing complete methods. First we consider the aspect of developing multi-level abstractions. Thereafter we consider the integration of products and processes.

\section{Multi-level Abstractions}

The concern in Information Systems is the development of the instant application. This approach is well suited to bespoke product development: domain knowledge, technical capability, and organizational functional/non-functional requirements are all brought together to build the new product. If there is any reuse then this involves picking up components of legacy products. The point is that development is concerned exclusively with products and not with abstractions of these.

The completeness principle proposes multi-level abstractions of products. By analogy with meta models of methods that abstract out common features of methods, it can be seen that application concepts can also be meta modeled, thus giving rise to an application abstraction. Extending this argument to pairs of levels, the completeness principle proposes multi-levels of application abstractions. From the perspective of the conceptualization principle, this can be understood as its application to levels above the traditional conceptual one.

ERP systems (ASA99) model the wider context of applications. Thus, if an organization wants to use an ERP system for materials management, then a general framework exists that must be customized to the specific needs of the organization. Additional functionality can be introduced by using adaptation features like ABAP of SAP. Further, ERP systems are defined at relatively low, operational levels of abstraction. However, the problem of the Information Systems community is different from that solved by ERP systems. This community gives primacy to 
conceptualization and abstractions and leaves the task of converting these into operational systems to downstream stages in the life cycle. Complete methods and the completeness principle addresses the conceptualization/abstraction concerns of the Information Systems community.

Enterprise modeling (Fox97, Fox 98, Lou95) is again different from developing abstractions of applications because of its emphasis on modeling the larger information context of the enterprise within which the system To-Be is embedded. It is not interesting per se to look at the information system to be developed which is only a fall out of enterprise modeling. In contrast, complete methods limit themselves to multi-level abstractions of information systems. As to whether or not enterprises can be usefully abstracted for each level of application abstraction is an open issue.

We can draw an analogy between product families and product lines on the one hand and method abstractions on the other. Consider a path from the root of the abstraction hierarchy to a leaf. A method on this path is a variant of another irrespective of whether it is above or below it in the hierarchy. In this sense, we obtain a method line that is analogous to product lines.

\section{Balancing Products with Processes}

The area of method engineering lays great emphasis on meta models (Har94, Gro97, Pra98). Depending on the nature of the meta model, we can instantiate methods that can build exclusively the application product, or the Information System Development process, or both of these. Irrespective of this, for the application engineer, the end result of the application of a method is the Information Systems product that can eventually be installed and operated upon. Even the $100 \%$ principle that lays emphasis on an exact representation of the real world does so in terms of the IS product: application concepts, inter-relationships between these, and constraints. Complete methods give equal emphasis to the IS product and the application process. It argues for an equal treatment to be given to process concepts, inter-relationships between these and constraints, heuristics etc.

The process model is represented in its full richness and variations as a dependency graph. This enables us to look at each individual method feature and its relationship with other features. There is a successor-predecessor relationship between method features. However, this relationship is controlled by the urgency and necessity properties. These properties specify the time delay in the relationship and also identify whether a feature must necessarily be enacted or is only a possibility, a choice.

The dependency graph looks similar to the notion of a map [Rol99]. However, the map is at a completely different level of abstraction. Its nodes are intentions and edges are strategies for fulfilling these intentions. This is in contrast to a dependency graph where nodes represent the capability of operating on given product elements and edges are dependencies of different types. Again, in a map, there is a successorpredecessor relationship between intentions and this relationship is controlled by different map topologies: bundles, multi-paths, etc. In contrast, a dependency graph uses the properties of dependencies to exercise control of what can/must be enacted next. It can be seen that there is no notion in a map corresponding to that of the property, urgency, of our dependencies. 


\section{Impact on Method Engineering}

The completeness principle introduces two new problems in method engineering, corresponding to the two aspects of multiple level of abstractions and application process models respectively. Consider the former. When a new method is to be engineered, its position in the abstraction hierarchy is to be determined. If it is a refinement of another method, then an appropriate relationship between the two is established. If it is an abstraction of another method, then it is to be placed at a higher level of refinement than this one. Finally, if none of these two conditions is satisfied then the new method is the root of a new abstraction hierarchy.

The organization of and retrieval from the method repository now becomes more complex than in traditional method engineering. Whereas earlier we were concerned with issues of keeping method descriptors or project characteristics in the repository, we now have to additionally move to a different kind of information. Descriptor/characteristic data only becomes relevant after the method is located in the abstraction hierarchy first. One way of handling this is to partition the repository into two parts, a meta method data part that contains abstraction hierarchy information and another, the method data part that contains descriptor/characteristic information. However, this is an open issue connected with the implementation of the completeness principle.

Now consider the issue of application process models. Any new method that we build must be engineered to accept information about the APM to be followed. We see two possible strategies. The first is the one we have adopted in this paper: generate the set of all possible application process models from which the desired one can be chosen. The second strategy is to produce the unique application process model that shall be used. Experience with the Map shows that the first strategy is perhaps better. One reason for this is the possibility of capturing variations in the set of models. However, we believe that this issue needs to be investigated further.

\section{Conclusion}

Traditional methods are limited in two ways (a) they fail to produce the application process model, and (b) they do not consider product abstractions. Complete methods attempt to address both these. The completeness principle provides:

Tight integration of the product and process aspects: Research in methods has pointed out the need to integrate product and process aspects of methods together. The completeness principle combines the existing 100\% and conceptualization principles to put this integration on a firm conceptual basis.

Better product fit: Products are to be embedded in organizational processes. The completeness principle requires methods to build the application process model associated with the product under development. This shall provide a basis to verify that the process model built is in conformity with the business process of the organization. The closer these two are to one another the more likely it shall be that the product shall smoothly fit in the organization. 
Support for process re-engineering: The ability to build the APM makes it possible to determine the extent of its fitness with organizational processes. Any differences between these can form the basis for informed debate on APM selection/process re-engineering.

\section{References}

(ASA99) ASAP World Consultancy and J. Blain et al, Using SAP R/3, Prentice Hall of India, 1999

(Fox97) Fox M.S. \& Gruninger M., On Ontologies and Enterprise Modelling, Intl. Conf. On Enterprise Integration Modelling, Italy, 1997

(Fox98) Fox M.S. \& Gruninger M., Enterprise Modelling, AI Magazine, 19, 3, 109 - 121, 1998

(Gri82) Griethuysen JJ van (ed.) Concepts and Terminology for the Conceptual Scheme and the Information Base, Publication Nr. ISO/TC97/SC5/WG3-N695, ANSI, New York

(Gro97) Grosz G., et al, Modelling and Engineering the Requirements Engineering Process: An Overview of the NATURE Approach, Requirements Engineering Journal, 2, 3, 115-131

(Har94) Harmsen F., et al, Situational Method Engineering for Information System Project Approaches, in Methods and Associated Tools for the Information Systems Life Cycle, Verrijn-Stuart and Olle (eds.), Elsevier, 169-194

(Hoe94) ter Hoefstede, Propoer AHM., van der Weide, Formal Description of a Conceptual Language for the Description and Manipulation of Information Models, Information Systems, 18(7) 489-523

(Lou95) Loucopoulos P., \& Kavakli E., Enterprise Modeling and the Teleological Approach to Requirements Engineering, IJCIS, 45 - 79, 1995

(Met93) MetaEdit version 1.2, MetaCase Consulting, Jyvaskyla, FInland

(Pra99) Prakash N., (1999) On Method Statics and Dynamics, Information Systems Journal, 24, 8, 613-637.

(Pra06) Prakash Naveen, On Generic Method Models, Requirements Engineering Journal, 11, 4, 221-237, 2006

(Rol99) Rolland C., Prakash N., and Benjamen A., A Multi-model View of Process Modelling, Requirements Engineering Journal, 4, 4, 169-187, 1999

(Rai03) Ralyté J; Deneckère R., Rolland C., Towards a Generic Model for Situational Method Engineering, Proc. CAiSE 2003, Eder J. \& Missikoff M. (eds.) LNCS 2681, Springer, $95-$ 110 . 